\title{
Improvement of Homogeneity and Aspect Ratio of Silicon Tips for Field Emission by Reactive-Ion Etching
}

\author{
Robert Damian Lawrowski, Christian Prommesberger, Christoph Langer, \\ Florian Dams, and Rupert Schreiner
}

Faculty of General Sciences and Microsystems Technology, OTH Regensburg, 93053 Regensburg, Bavaria, Germany

Correspondence should be addressed to Robert Damian Lawrowski; robert.lawrowski@oth-regensburg.de

Received 16 February 2014; Accepted 9 March 2014; Published 6 April 2014

Academic Editor: Feng Zhao

Copyright (c) 2014 Robert Damian Lawrowski et al. This is an open access article distributed under the Creative Commons Attribution License, which permits unrestricted use, distribution, and reproduction in any medium, provided the original work is properly cited.

\begin{abstract}
The homogeneity of emitters is very important for the performance of field emission (FE) devices. Reactive-ion etching (RIE) and oxidation have significant influences on the geometry of silicon tips. The RIE influences mainly the anisotropy of the emitters. Pressure has a strong impact on the anisotropic factor. Reducing the pressure results in a higher anisotropy, but the etch rate is also lower. A longer time of etching compensates this effect. Furthermore an improvement of homogeneity was observed. The impact of uprating is quite low for the anisotropic factor, but significant for the homogeneity. At low power the height and undercut of the emitters are more constant over the whole wafer. The oxidation itself is very homogeneous and has no observable effect on further variation of the homogeneity. This modified fabrication process allows solving the problem of inhomogeneity of previous field emission arrays.
\end{abstract}

\section{Introduction}

In vacuum microelectronic devices the field emission (FE) electron sources have advantages compared to classical thermionic cathodes. They offer no dissipation of energy in the medium (vacuum) and high radiation tolerance and work with high operation frequency [1]. The electron sources could be used in sensor systems, miniaturized microwave amplifier tubes, cathodes in electron optic systems (e.g., scanning electron microscope (SEM), scanning tunneling microscope (STM), transmission electron microscopy (TEM)), and high power $\mathrm{THz}$ sources as well as compact and fast-switching $\mathrm{X}$-ray sources [2]. The cathode of the electron source is the most important and critical component of such devices. Small variation in emitter geometry leads to an inhomogeneity of emission. The field emission characteristics depend especially on the width of the potential barrier at the electrically conductive surface, which the electrons must tunnel through [3]. High electric fields reduce the width of this barrier (Figure 1). Nanostructures allow delivering these required fields microscopically, due to the locally enhanced field at the tip of the emitter. The field enhancement factor $\beta$
(1) which is defined by the ratio of microscopic $E_{\text {micro }}$ to macroscopic field $E_{\text {macro }}$ describes this relation [4]. Therefore, lower macroscopic field is necessary for tunneling:

$$
\beta=\frac{E_{\text {micro }}}{E_{\text {macro }}} \approx \frac{h}{r} .
$$

A possible approximation for $\beta$ is the ratio of height $h$ of the emitter to the radius $r$ of the tip. Consequently, fluctuations in height and tip radius vary strongly the $\beta$ factor. Further enhanced fabrication techniques are required to replace typical cathodes in actual applications and allow novel vacuum devices.

By investigation of the influence of RIE parameters (pressure and power) on the geometry and aspect ratio of silicon emitter a chance of homogeneity can be observed.

\section{Materials and Methods}

2.1. Fabrication Process. Isotropic wet or dry and anisotropic dry etching are usual fabrication techniques for silicon (Si) microstructures. For reproducible emitters with high $\beta$-factor 


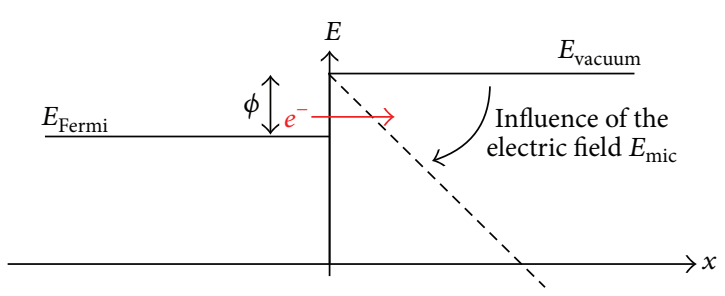

FIGURE 1: Influence of an enhanced electric field $E_{\text {micro }}$ on the surface barrier and the tunneling of electrons.

and current carrying capacity, we use an anisotropic dry etching followed by a wet thermal oxidation [5] (Figure 2).

As bulk material $100 \mathrm{~mm}$ p-type silicon wafers with (100) orientation, boron doping, and a resistivity of 3.7$4.2 \Omega \mathrm{cm}$ are used. P-doped Si-FE-cathodes show FE-current saturation, which leads to very good current stabilization [6]. For masking a wet thermal oxide of $700 \mathrm{~nm}$ is grown on the substrate at $1000^{\circ} \mathrm{C}$ (Figure 2(a)). The position of the tips is defined by a photolithographic transfer of disks with a $3 \mu \mathrm{m}$ diameter and triangular pitch of $20 \mu \mathrm{m}$ into a photoresist (AZ5214) (Figure 2(b)). Anisotropic reactive-ion etching in an Oxford Plasmalab 80Plus transfers this adjustment to the $\mathrm{SiO}_{2}$ layer (Figure $2(\mathrm{c})$ ). It uses $\mathrm{CHF}_{3}$ and $\mathrm{O}_{2}$ as process gases. To achieve the requested shape of the emitter another RIE process step is necessary. A mixture of $\mathrm{SF}_{6}$ and $\mathrm{O}_{2}$ etches bulk $\mathrm{Si}$ with the $\mathrm{SiO}_{2}$ discs as mask (Figure 2(d)). The anisotropy and so the geometry can be adjusted by the gas flows, chamber pressure, and RF power [7]. The remaining $\mathrm{Si}$ is oxidized thermally at $940^{\circ} \mathrm{C}$ for the final sharpening of the emitters (Figure 2(e)). The entire $\mathrm{SiO}_{2}$ is removed in the last step by wet chemical etching with a HF mixture (BOE 7:1) (Figure 2(f)).

To get significant investigation on the homogeneity each of the twelve chips on a wafer contains six arrays with a different number of tips $(1,7,91,271,547$, and 1141).

2.2. Measurement Techniques and Experimental Design. For the investigation of the influence of individual fabrication steps on the emitter, after each process step, some selected tips were observed by a SEM (JEOL 6510) (Figure 3). For a significant conclusion of the homogeneity eight tips on each array were scanned on every corner of the hexagonal layout and two in the middle of the array (Figure 4(a)). Two of three chips on each quarter of a wafer were investigated (Figure 4(b)).

The undercut $r_{\|}$of the $\mathrm{SiO}_{2}$-mask, due to the RIE step, was determined by SEM. The etch depth $r_{\perp}$ was measured with a KLA Tencor P16 profilometer. By this way, the anisotropic factor (2), which impacts the homogeneity and geometry of the emitters, was determined for diverse powers and pressures at the RIE step:

$$
f=1-\frac{r_{\|}}{r_{\perp}}
$$

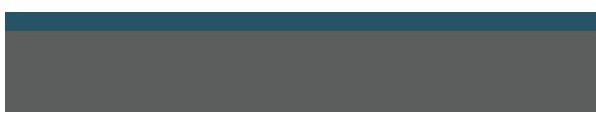

(a) Oxidation

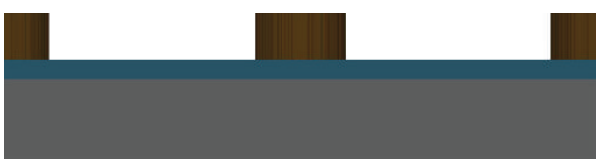

(b) Photolithography

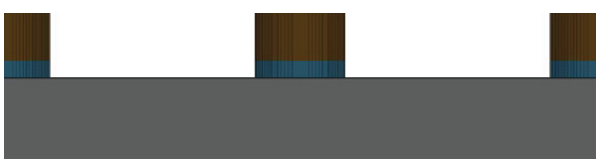

(c) $\mathrm{RIE}$ of $\mathrm{SiO}_{2}$

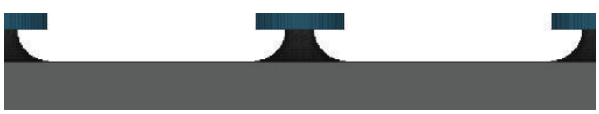

(d) RIE of Si

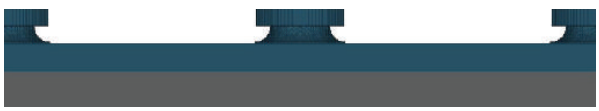

(e) Sharpening Oxidation

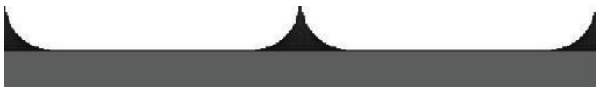

(f) Si-tip-emitter

FIGURE 2: Schematic of the structured cathode fabrication process (simulated with IntelliSuite). (a) Thermal growth of $\mathrm{SiO}_{2}$ layer. (b) Photolithography with AZ5214. (c) Transferring of the structures into $\mathrm{SiO}_{2}$ by reactive-ion etching. (d) Reactive-ion etching of the bulk Si. (e) Sharpening of the tips by wet thermal oxidation. (f) Wet chemical removal of $\mathrm{SiO}_{2}$.

TABLE 1: Anisotropy factor $f$ depending on the etching parameters (pressure and power). Symbols in brackets belong to Figure 6.

\begin{tabular}{lccc}
\hline & 50 mTorr & 70 mTorr & 90 mTorr \\
\hline $90 \mathrm{~W}$ & $0.58(\bigcirc)$ & $0.55(\square)$ & $0.43(\diamond)$ \\
$120 \mathrm{~W}$ & $0.61(\triangle)$ & $0.53(\nabla)$ & - \\
$150 \mathrm{~W}$ & $0.65(\triangleright)$ & $0.51(\triangleleft)$ & - \\
\hline
\end{tabular}

\section{Results and Discussion}

The power of plasma is varied from 90 to $150 \mathrm{~W}$ and the pressure from 50 to 90 mTorr, resulting in 9 several parameter sets (Table 1) with different anisotropy (Figure 5). The etching time amounts to $60 \mathrm{~s}$. A full undercut of the $\mathrm{SiO}_{2}$ disks is caused by a pressure of 90 mTorr at high power $(120 \mathrm{~W}$ or $150 \mathrm{~W})$.

3.1. Effect of RIE Parameters on the Geometry. The pressure has a strong effect on the factor of anisotropy. The reduction of pressure increases significantly the anisotropic factor (Figure 6(a)). Lower pressure causes fewer ions in the plasma, which are able to collide with each other at the same power in the chamber. Hence the ions are more directed perpendicular 


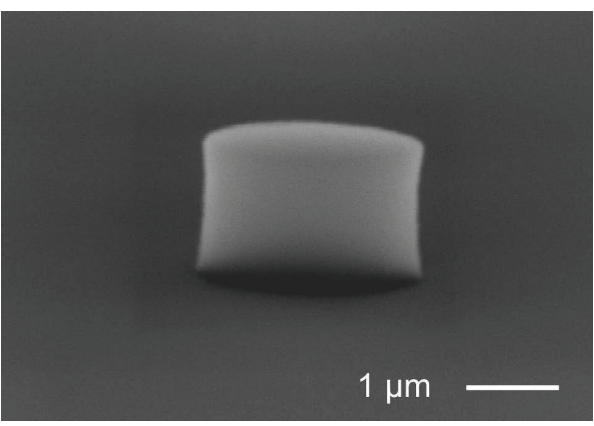

(a) Photolithography

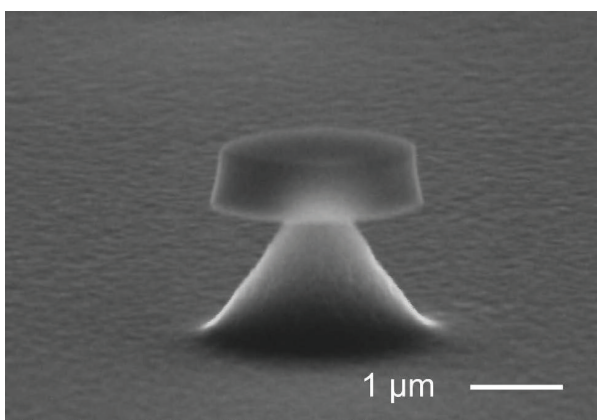

(c) Si-RIE-etching

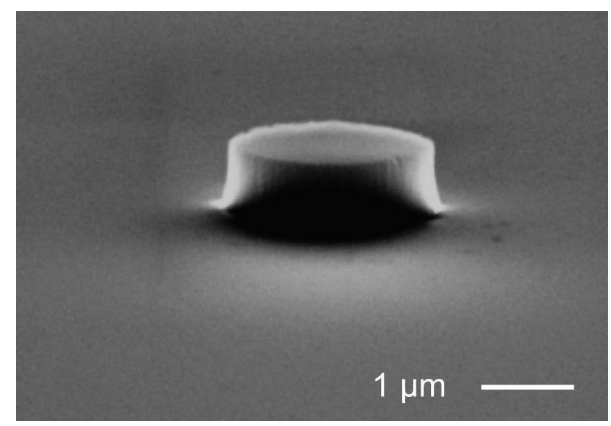

(b) $\mathrm{SiO}_{2}$-RIE-etching

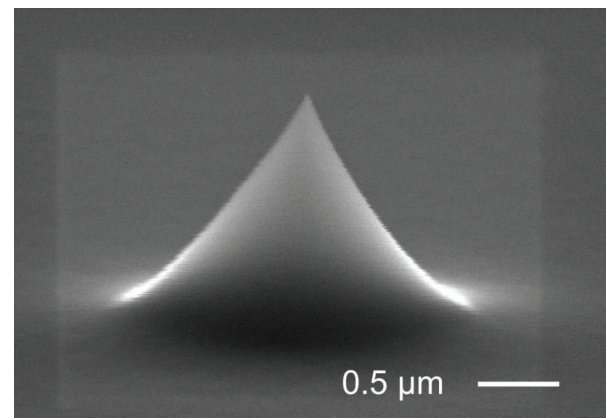

(d) Si-tip-emitter

Figure 3: SEM images of the structured cathode fabrication process. (a) Developed photoresist. (b) Patterned $\mathrm{SiO}_{2}$ disks. (c) Etched bulk Si. (d) Final emitter.

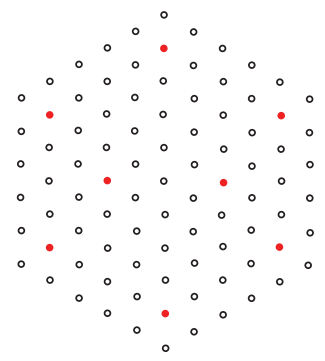

(a)

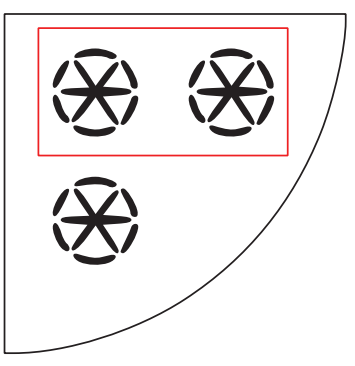

(b)
FIGURE 4: Schematic position of the investigated (a) tips in an array ( 8 of 91) and (b) chips on each quarter ( 2 of 3 )

to the target. Therefore the anisotropic factor is high at low pressures and vice versa.

By increasing the power, the energy of the ions in the chamber is rising. At low pressure (blue line in Figure 6(b)) they are more able to impact the target without other hits. The anisotropic factor is rising, too. The higher the pressure is, the more reactive the molecules $\mathrm{SF}_{6}$ are in the plasma. That means that there are more possible collisions on the way, because of the higher number of ions in the chamber. Moreover the effect of the chemical reaction is stronger and the directions of the impacting ions are more distributed, so the etching result is less anisotropic. This results in a lower anisotropy factor, while energy is higher (green line in Figure 6(b)).
Due to a higher anisotropy of etching, the aspect ratio of the tip can be maximized. This causes a larger $\beta$-factor.

\subsection{Investigation on Homogeneity and Measurement Results.} Changing power and pressure causes a variation of etch rates within the radius of the wafer, which is the homogeneity of the unit (Figure 7). SEM images are shown in Figure 8. Furthermore, low pressure ( 50 mTorr) results in quite homogeneous etching, regarding height of emitters and undercut of disks (Figures 7(c) and 7(d)). There are fewer ions in the plasma, due to low pressure in the chamber. The scattering of the ions on other particles and $\mathrm{SiO}_{2}$ disks on the wafer is lower, leading to a more controlled and homogeneous process. However the etch rate is lower. This effect can be compensated by a longer time $(1.5 \mathrm{x}-2.0 \mathrm{x})$ of etching.

The final oxidation thickness is dependent on the remaining diameter of the $\mathrm{Si}$ under the $\mathrm{SiO}_{2}$. The thermal wet oxidation is very homogeneous (a variation of less than $4 \%$ over the whole wafer). They have no observable effect on further differences of the homogeneity of the etched emitters.

Hence, with a lower pressure, it is possible to produce quite homogeneous cathodes with a high anisotropic factor across the wafer (Figures 8(c) and 8(d)).

FE measurements were performed on tip arrays, which were etched at low pressure (50 mTorr) and with an increased power of $120 \mathrm{~W}$ in order to get a high anisotropy factor (Figure 6). The height was around $1 \mu \mathrm{m}$ with a radius of $<40 \mathrm{~nm}$ and a tip-to-tip distance of $20 \mu \mathrm{m}$ (Figure 9). 


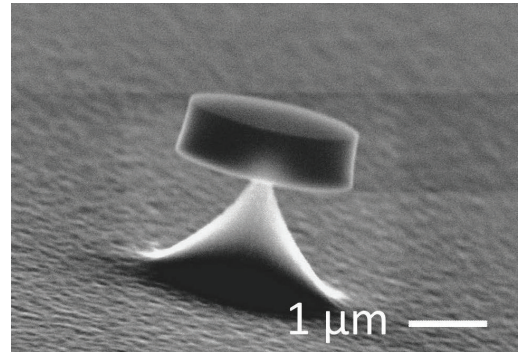

(a)

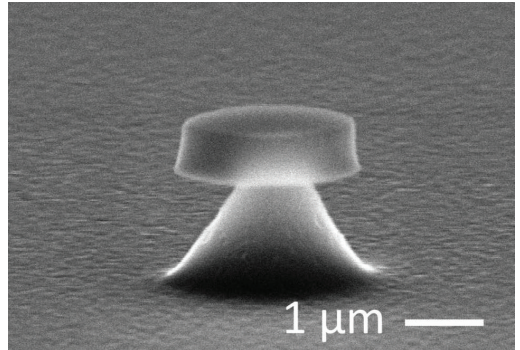

(b)

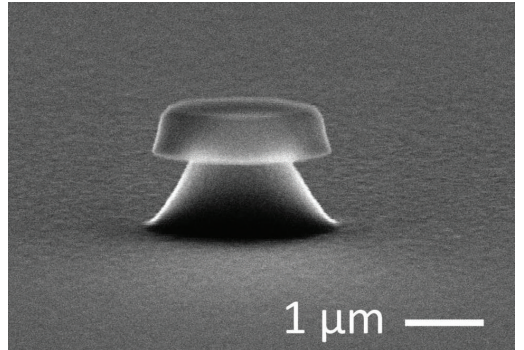

(c)

FIGURE 5: SEM images of tips after the Si etching with three different anisotropy factors: (a) $f=0.4$, (b) $f=0.5$, and (c) $f=0.6$.

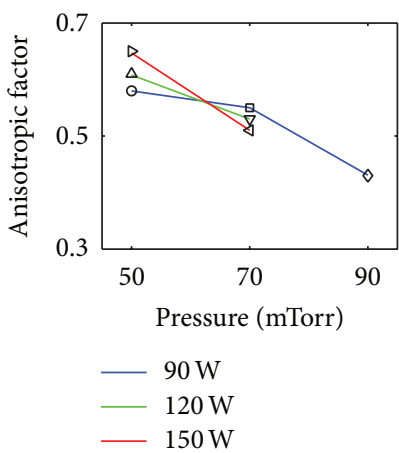

(a)

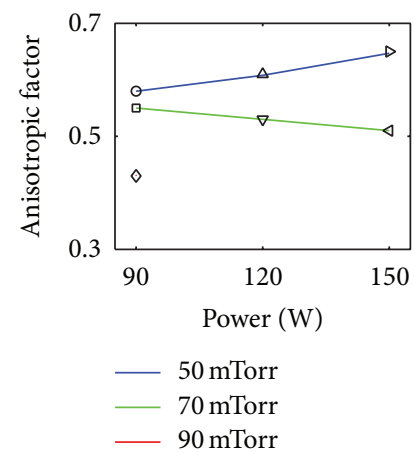

(b)

FIGURE 6: Dependence of the anisotropic factor on pressure (a) and power (b).

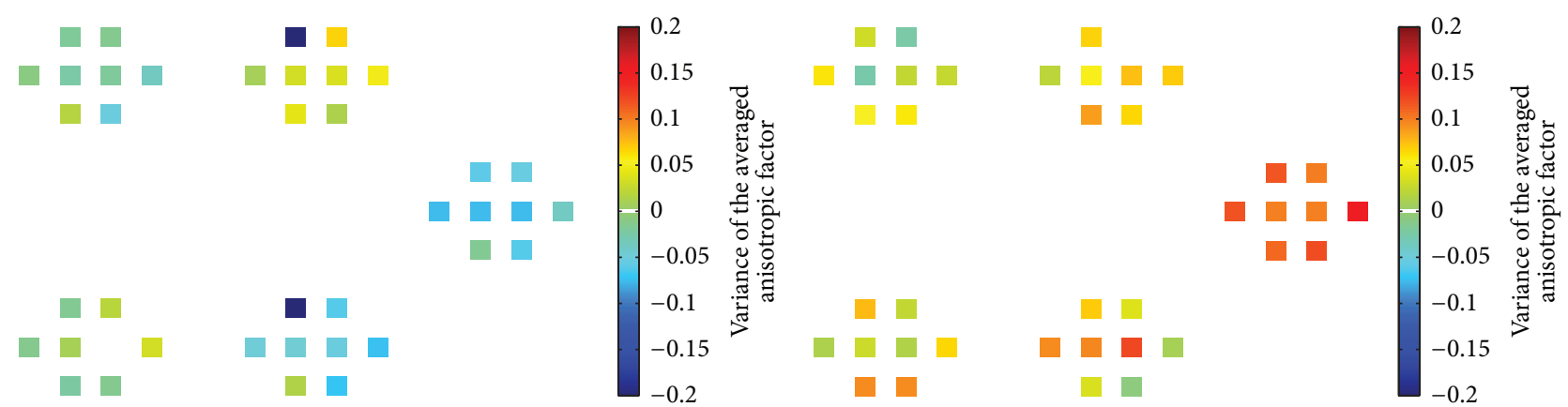

(a) Arrays near wafer center (rms deviation: 0.063 ) (mean value: -0.029 )

(b) Arrays near wafer edge (rms deviation: 0.075) (mean value: 0.061)

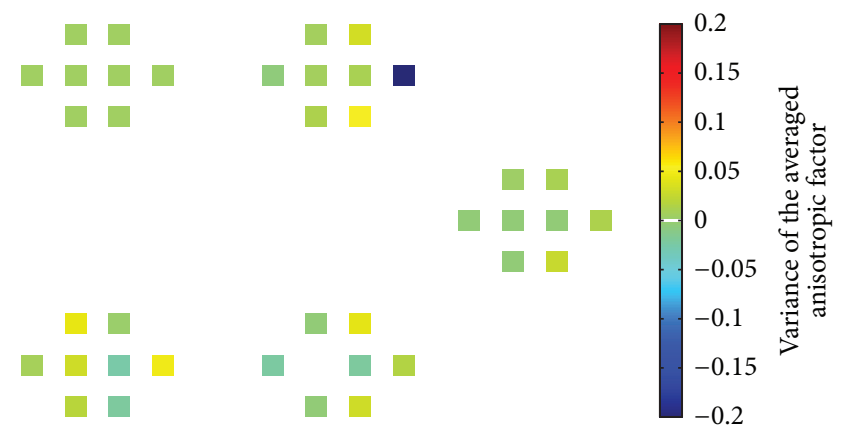

(c) Arrays near wafer center (rms deviation: 0.048) (mean value: 0.016)

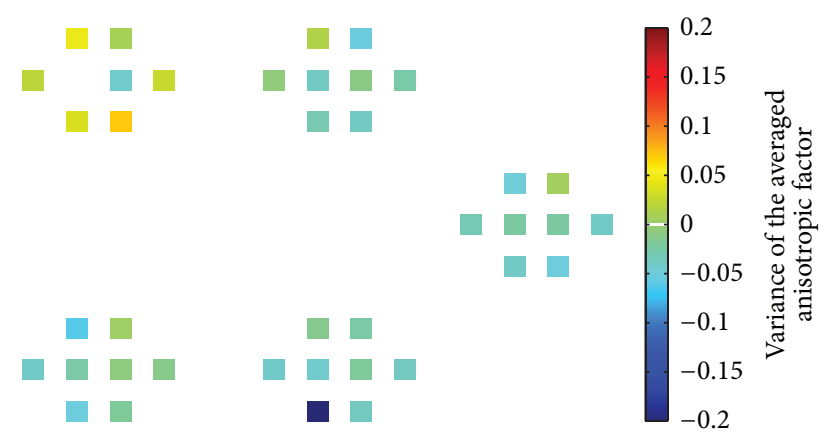

(d) Arrays near wafer edge (rms deviation: 0.049) (mean value: -0.023 )

FiguRE 7: Variation of the anisotropic factor from the averaged value of anisotropy on the quarter at different etch parameters: (a) and (b) 90 mTorr, 60 s, $90 \mathrm{~W}$, (c) and (d) 50 mTorr, 90 s, 90 W. Values in brackets are the root mean square deviation and the arithmetic mean of the variation. 


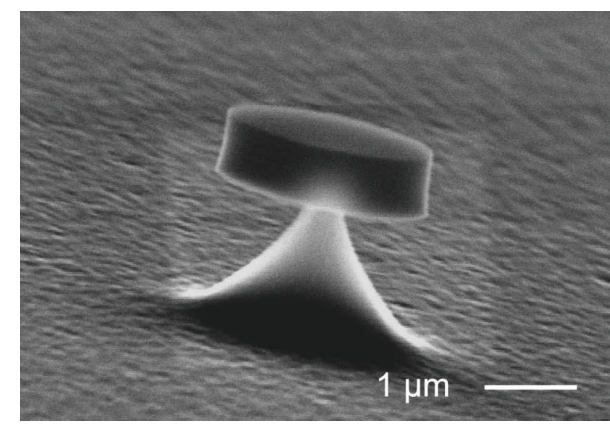

(a) Near wafer center

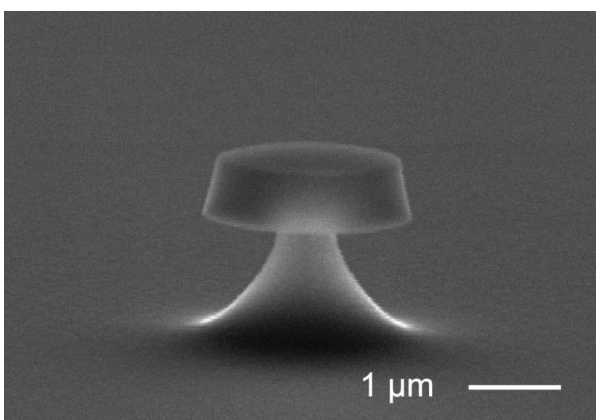

(c) Near wafer center

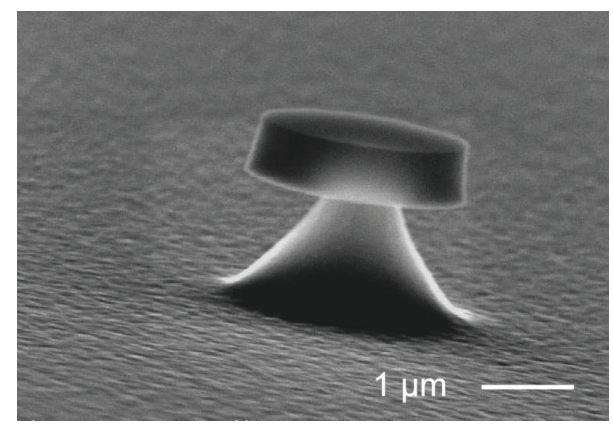

(b) Near wafer edge

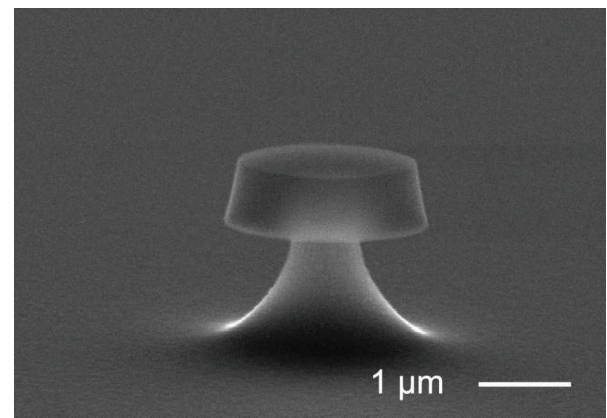

(d) Near wafer edge

FIgure 8: SEM images of the emitters after RIE of Si at different etching parameters: (a) and (b) 90 mTorr, 60 s, 90 W, (c) and (d) 50 mTorr, $90 \mathrm{~s}, 90 \mathrm{~W}$.

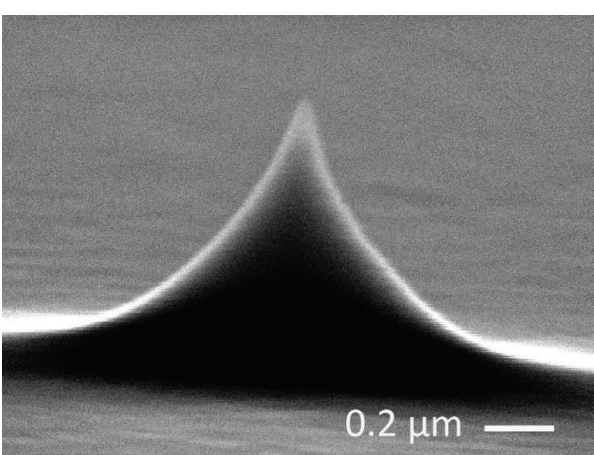

(a)

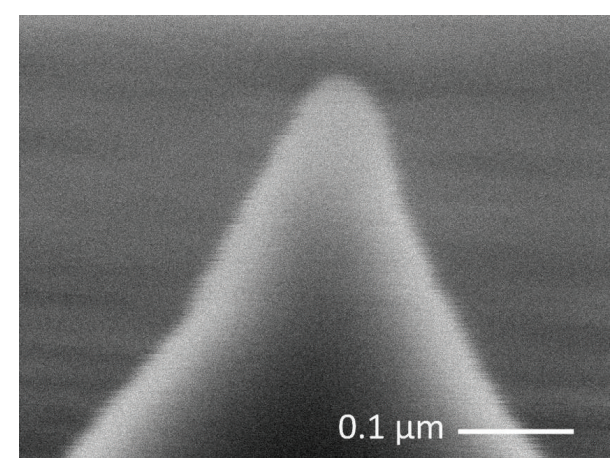

(b)

FIGURE 9: SEM images of emitters measured at the investigation: (a) with a height of $0: 9 \mu \mathrm{m}$ and (b) with a radius of $40 \mathrm{~nm}$.

A comprehensive investigation of the field emission properties of these structures is given in [6]. The array with 547 emitters showed an integral FE-current up to $10^{-7} \mathrm{~A}$ at an electric field of $50 \mathrm{~V} / \mu \mathrm{m}$. An on-set-field of around $40 \mathrm{~V} / \mu \mathrm{m}$ for a FE-current of $1 \mathrm{nA}$ was measured. Furthermore the optimization in the RIE process results in a homogeneous emission over the entire array with almost $99 \%$ efficiency [6].

The investigation of the anisotropy over the wafer (part 3.2) proves that a low pressure of around 50 mTorr results in a good homogeneity of the emitters over the complete wafer area. The FE measurement showed a good homogeneity of all individual emitters within an array, too.

\section{Conclusion and Outlook}

The dependence of anisotropy on the etching parameters (pressure and power) of RIE was investigated. Higher pressure increases the lateral etching rate and results in lower anisotropy. The influence of power on the anisotropic factor depends on the pressure. Low pressure causes higher anisotropy with a less scattering of the anisotropic factor. The homogeneity over the entire wafer was improved and due to the higher anisotropy a high aspect ratio of the tips was realized. With adjusted power an optimized etching step was achieved. The field emission investigation shows 
homogeneous emission of the emitters, which were fabricated with this enhanced process. This opens the possibility to build homogeneous cathodes, which are able to carry stable current for advanced vacuum microelectronic devices.

\section{Conflict of Interests}

The authors declare that there is no conflict of interests regarding the publication of this paper.

\section{Acknowledgments}

The underlying project was founded by the German Federal Ministry of Education and Research with sponsorshipnumber 03FH004PX2. The authors of this paper assume resposibility for its content. The authors would like to thank the team for the support.

\section{References}

[1] R. Schreiner, F. Dams, C. Prommesberger et al., "Silicon-based integrated field emission electron sources for sensor application," in Proceedings of the 24th International Vacuum Nanoelectronics Conference (IVNC '11), pp. 19-20, July 2011.

[2] R. Schreiner, C. Prommesberger, C. Langer et al., "Highly uniform and stable electron field emission from b-doped sitip arrays for applications in integrated vacuum microelectronic devices," in Proceedings of the 25th International Vacuum Nanoelectronics Conference (IVNC '12), pp. 1-2, 2012.

[3] R. H. Fowler and L. Nordheim, "Electron emission in intense electric fields," Proceedings of the Royal Society of London A, vol. 119, no. 781, pp. 173-181, 1928.

[4] C. Langer, C. Prommesberger, F. Dams, and R. Schreiner, "Theoretical investigations into the field enhancement factor of Silicon structures," in Proceedings of the 25th International Vacuum Nanoelectronics Conference (IVNC '12), pp. 148-149, July 2012.

[5] F. Dams, A. Navitski, C. Prommesberger et al., "Homogeneous field emission cathodes with precisely adjustable geometry fabricated by Silicon technology," IEEE Transactions on Electron Devices, vol. 59, no. 10, pp. 2832-2837, 2012.

[6] P. Serbun, B. Bornmann, A. Navitski et al., "Stable field emission of single b-doped si tips and linear current scaling of uniform tip arrays for integrated vacuum microelectronic devices," Journal of Vacuum Science \& Technology B: Microelectronics and Nanometer Structures, vol. 31, no. 2, Article ID 02B101, 2013.

[7] R. Legtenberg, H. Jansen, M. de Boer, and M. Elwenspoek, "Anisotropic reactive ion etching of Silicon using $\mathrm{SF}_{6} / \mathrm{O}_{2} / \mathrm{CHF}_{3}$ gas mixtures," Journal of the Electrochemical Society, vol. 142, no. 6, pp. 2020-2028, 1995. 

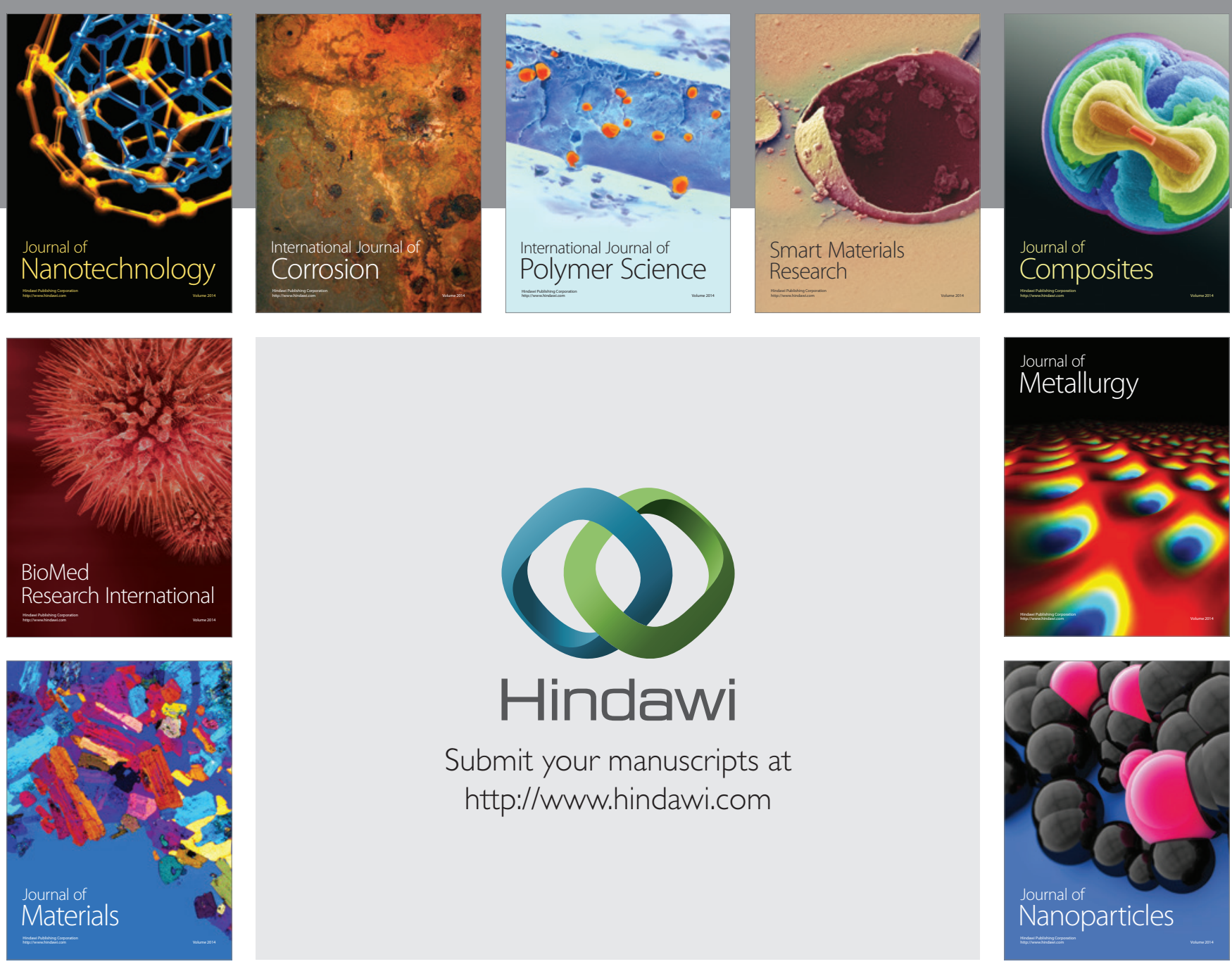

Submit your manuscripts at http://www.hindawi.com
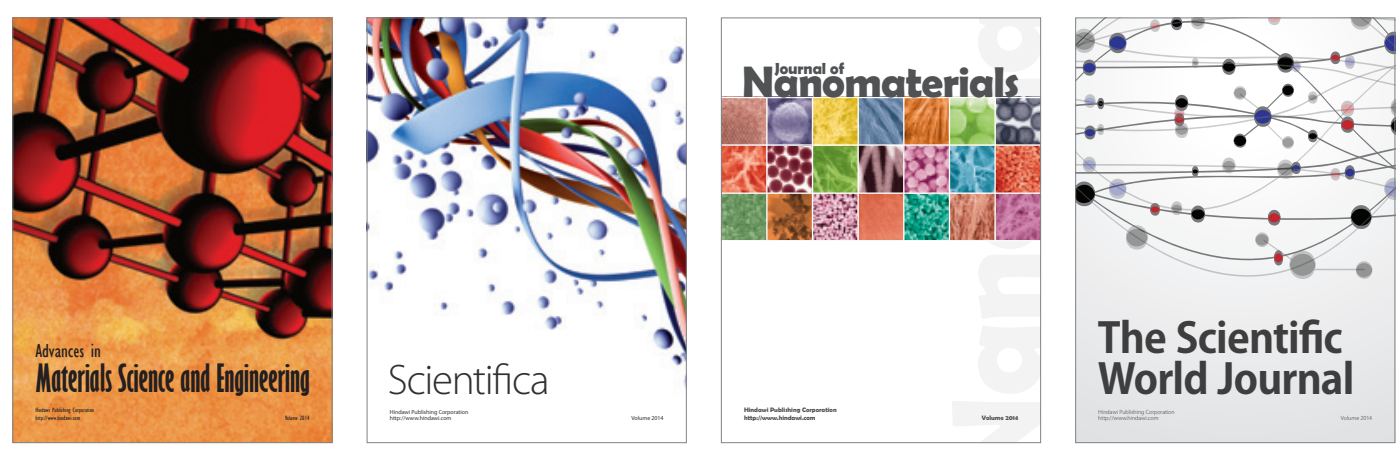

\section{The Scientific World Journal}
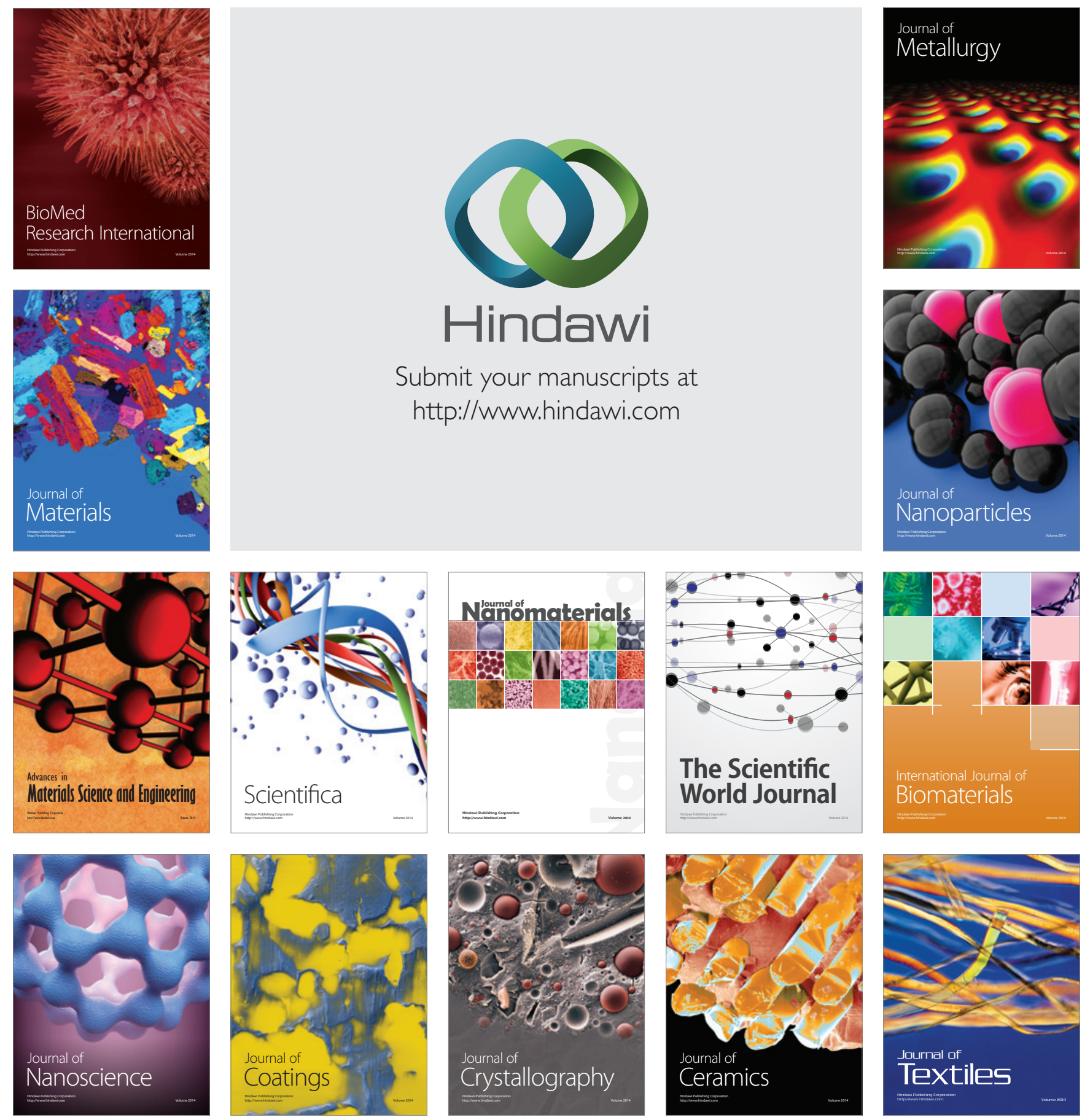